\title{
Confirmed Thromboembolic Venous Disease and Clinical Probability Scores: A Transversal Study about 74 Cases Collected in the Cardiology Department of the Aristide Le Dantec University Hospital Center of Dakar
}

\author{
Sidy Lamine Sy ${ }^{1 *}$, Simon Joël Manga², Sérigne Mor Beye ${ }^{3}$, El Hadj Mbacké Sarr ${ }^{1}$ \\ ${ }^{1}$ Cardiology Department, National University Hospital of Fann, Dakar, Senegal \\ ${ }^{2}$ Cardiology Department, De La Paix Hospital, Ziguinchor, Senegal \\ ${ }^{3}$ Cardiology Department, Saint-Louis Regional Hospital, Saint-Louis, Senegal \\ Email: *lemzodiamono82@hotmail.com
}

How to cite this paper: Sy, S.L., Manga, S.J., Beye, S.M. and Sarr, El H.M. (2021) Confirmed Thromboembolic Venous Disease and Clinical Probability Scores: A Transversal Study about 74 Cases Collected in the Cardiology Department of the Aristide Le Dantec University Hospital Center of Dakar. Case Reports in Clinical Medicine, 10, 373-380.

https://doi.org/10.4236/crcm.2021.1011046

Received: October 4, 2021

Accepted: November 6, 2021

Published: November 9, 2021

Copyright $\odot 2021$ by author(s) and Scientific Research Publishing Inc. This work is licensed under the Creative Commons Attribution International License (CC BY 4.0).

http://creativecommons.org/licenses/by/4.0/ (c) (i) Open Access

\begin{abstract}
Introduction: Venous thromboembolic disease (VTED), associating deep vein thrombosis and pulmonary embolism, represents a major public health issue. The objective of our work is to correlate confirmed VTED with clinical probability scores using elements of interview and clinical examination. Methods: This was a retrospective study from January 1, 2012 to October 27, 2013. Venous thromboembolic disease was diagnosed by lower limb venous Doppler ultrasound for deep vein thrombosis and thoracic CT angiography for pulmonary embolism. Results: Our series included 74 cases of venous thromboembolic disease including 42 cases of deep vein thrombosis and 29 cases of pulmonary embolism. The average age was $48.5 \pm 15.9$ years. The sex ratio was 0.72 . The patients came from the outpatient clinic in $67.57 \%$ of cases. The Wells score for pulmonary embolism showed excellent performance in the "Surgery/Cancer" subgroup where the low probability was zero. The revised Geneva score for pulmonary embolism, showing the same proportions of low (14.2\%) and intermediate (85.7\%) probability, did not discriminate the subgroup of patients with underlying heart disease from the one from a surgical or carcinological environment. Conclusion: Clinical probability scores are more suitable in surgical and oncological settings than in medical settings.
\end{abstract}

\section{Keywords}

Venous Thromboembolic Disease, Wells Score, Geneva Score, Dakar, Senegal 


\section{Introduction}

Venous thromboembolic disease (VTED) is a concept associating two inseparable entities, deep venous thrombosis (DVT) and its immediate complication, pulmonary embolism (PE) [1].

DVT is a more or less extensive obliteration of a vein, by the constitution and development in the lumen of a thrombus, most often of the fibrino-cruoric type. $\mathrm{PE}$ is a sudden obliteration of the trunk or a branch of the pulmonary artery by an embolus, most often from a DVT of the lower extremities [2].

MVTE represents a major public health issue. In fact, in industrialized countries, it is the 4th leading cause of death and the 3rd leading cause of cardiovascular death behind myocardial infarction and stroke.

Its incidence is often underestimated because it can be asymptomatic, misdiagnosed or not reported as the underlying cause of death.

The clinical signs usually described have variable sensitivities and specificities making the diagnosis risky. Faced with this clinical difficulty, clinical probability scores and decisional algorithms have been established in order to best guide practitioners in the choice of additional examinations, currently allowing great precision diagnosis with minimal cost and risk to the patient.

The diagnosis is based on the venous EchoDoppler of the lower limbs and the $\mathrm{CT}$ angiography which are expensive examinations and which are not very accessible in our regions.

The clinical likelihood assessment is the first step in treating a patient with suspected VTE. This assessment is based either on the clinician's own conviction or on clinical probability scores (SPC). The two possibilities are equivalent even if the reproducibility of the scores is slightly higher.

The revised Geneva score as well as the Wells score involves elements of the interview and clinical examination, while others require measurement of transcutaneous oxygen saturation (Charlotte's rule) or performance (and interpretation) of a chest X-ray (Miniati score, original version of the Geneva score) or arterial gas measurement (original version of the Geneva score).

In Africa, epidemiological data on VTED are scarce and difficult to collect.

The study from Samaladougou, Burkina Faso, found an average performance of these scores in pulmonary embolism [3].

No study has been devoted to it in Senegal.

The objective of our work is to correlate confirmed MVTE with clinical probability scores using interview's elements and physical examination.

\section{Patients and Methods}

We had conducted a retrospective study carried out at the cardiology clinic of the Aristide Le Dantec Hospital (HALD) from January 1, 2012 to October 27, 2013. We had recruited patients hospitalized in our department for confirmed MVTE.

All patients hospitalized and treated for pulmonary embolism and/or deep 
venous thrombosis confirmed by thoracic CT angiography and venous Doppler ultrasound of the lower limbs respectively were included.

We did not include in the study patients whose records were unusable and suspected cases of pulmonary embolism or deep vein thrombosis not confirmed by $\mathrm{CT}$ angiography or venous echoDoppler of the limbs lower.

The data collection base consisted of hospitalized patient records and the parameters studied concerned socio-demographic data (age, gender, socio-economic level, consultation time), hemodynamic constants, functional signs as well as clinical examination data. The results of additional examinations, the factors favoring the risk of the occurrence of VTED, treatment and hospital development were also studied.

The revised Geneva and Wells clinical scores as diagnostic tests were dichotomized as follows:

- Geneva revised score: Low clinical probability if score is between 0 and 3; Moderate clinical probability if score is between 4 and 10; High clinical probability if score $>11$.

- Wells score: Low clinical probability if score $<2$; Moderate clinical probability if score is between 2 and 6 ; Clinical probability if score $>6$.

Venous lower extremity echoDoppler and chest CT angiography were the reference exams.

The data collected was analyzed using Epi Info software version 7.1.2.0.

The descriptive study was carried out with the calculation of frequencies and proportions for the qualitative variables and the calculation of averages and standard deviations for the quantitative variables.

We ensured that the confidentiality of study participants was guaranted by using personal identification numbers on data collection forms instead of names. Personal identifiers will not be included in study reports. All study records will be kept confidential.

\section{Results}

The overall prevalence relative to the number of hospitalized patients $(\mathrm{N}=1834)$ during the same period was $4 \%$, or $1.7 \%$ for PE and $2.4 \%$ for DVT. More than half of the patients (42) or $56.75 \%$ had DVT, 39.18\% (29) had PE. Only $4.05 \%$ (3) had an associated form.

There was an overall female predominance of MVTE: 43 patients (58.10\%) or a sex ratio of 0.72 .

Over all of our patients, $83.78 \%$ (62 patients) had at least one risk factor for VTE. The most frequent risk factor found in our population for VTE was prolonged immobilization (Table 1) with 24 cases (32.43\%), followed by age $>60$ years with 23 cases, or $31 \%$. Seventeen cases of adiposity were found, or $23 \%$.

Almost all of the patients hospitalized for DVT had an intermediate or high probability according to the Wells score before confirmation with $80 \%$ high probability. All patients hospitalized for DVT with underlying heart disease and 
Table 1. Frequency of risk factors for VTED.

\begin{tabular}{lcc}
\hline \multicolumn{1}{c}{ Risk factors } & Number of cases & Percentage (\%) \\
\hline Prolonged immobilization & 24 & 32 \\
Age $>60$ ans & 23 & 31 \\
Adiposity (BMI $\geq 30)$ & 17 & 23 \\
Pregnancy and Postpartum & 15 & 12 \\
Recent surgery & 9 & 12 \\
Past history of Deep vein thrombosis & 9 & 11 \\
and pulmonary embolism & 8 & 9,5 \\
Oral contraceptives & 7 & 4 \\
Malignant neoplasia & 3 & \\
Limbs trauma & & \\
\hline
\end{tabular}

those arriving from a surgical or oncological setting had a high probability (Table 2).

Regarding the other patients coming from the outpatient and emergency department, $72.7 \%$ had a high probability, while $27.2 \%$ had an intermediate probability.

As for PE, according to Wells' score, it was found that half of the cases of PE had an intermediate probability. Only 04 patients or $12.5 \%$ had a high probability. More than a third of patients (37\%) had a low probability. In the subgroup of patients with PE and coming from a surgical or carcinologic setting, all had an intermediate $(57.1 \%)$ or high $(42.8 \%)$ one. The subgroup with a heart disease underlying had $28.5 \%$ of low probability. More than half of the patients hospitalized for PE from the outpatient or emergency department, or $52.6 \%$, had a low probability according to the Wells score (Table 3 ).

According to the revised Geneva score, 3 in 4 PE cases (75\%) had an intermediate probability. The low and high probabilities were $18.75 \%$ and $6.25 \%$, respectively. The subgroup of patients with PE from a surgical or oncological setting and those with underlying heart disease had the same probability of PE occurrence according to the revised Geneva score. Indeed, there was no high probability, the majority of patients having an intermediate one with $85.7 \%$. As for patients hospitalized for PE coming from the outpatient clinic or the emergency department, almost one in 4 cases, or $26.3 \%$, had a low probability and only $5.2 \%$ had a high probability (Table 4 ).

\section{Discussion}

Our series reports a MVTE prevalence of 4\%, including 2.7\% DVT and 1.4\% PE in 22 months. This hospital prevalence is clearly increasing compared to those of previous studies. This increase in the prevalence of the disease can be explained in part by the awareness of practitioners but above all thanks to the considerable advances in the field of imaging. 
Table 2. The clinical probability of the Wells score of DVT according to the origin of the patients.

\begin{tabular}{cccc}
\hline $\begin{array}{c}\text { Clinical } \\
\text { probability }\end{array}$ & Origin of patients & $\begin{array}{c}\text { Number of cases } \\
(\mathbf{n}=\mathbf{4 5})\end{array}$ & $\begin{array}{c}\text { Percentage } \\
(\%)\end{array}$ \\
\hline \multirow{2}{*}{ Intermediaite } & Cardiology & 0 & 0 \\
Surgery/Cancerology & 0 & 0 \\
High & Others & 9 & 27.2 \\
\hline & Surgery/Cancerology & 2 & 100 \\
& Others & 10 & 100 \\
\hline
\end{tabular}

Table 3. The clinical probability of the Wells score of PE according to the origin of the patients.

\begin{tabular}{cccc}
\hline $\begin{array}{c}\text { Clinical } \\
\text { probability }\end{array}$ & Origin of patients & $\begin{array}{c}\text { Number of cases } \\
(\mathbf{n}=32)\end{array}$ & $\begin{array}{c}\text { Percentage } \\
(\%)\end{array}$ \\
\hline \multirow{2}{*}{ Low } & Cardiology & 2 & 28.5 \\
& Surgery/Cancerology & 0 & 0 \\
Others & 10 & 52.6 \\
\hline \multirow{2}{*}{ Intermediate } & Cardiology & 3 & 42.8 \\
& Surgery/Cancerology & 4 & 57.1 \\
& Others & 7 & 36.8 \\
\hline High & Cardiology & 2 & 28.5 \\
& Surgery/Cancerology & 3 & 42.8 \\
& Others & 2 & 10.5 \\
\hline
\end{tabular}

Table 4. The clinical probability of the revised Geneva score of the PE according to the origin of the patients.

\begin{tabular}{cccc}
\hline $\begin{array}{c}\text { Clinical } \\
\text { probability }\end{array}$ & $\begin{array}{c}\text { Origin of } \\
\text { patients }\end{array}$ & $\begin{array}{c}\text { Numbre of } \\
\text { cases }(\mathbf{N}=32)\end{array}$ & Percentage (\%) \\
\hline \multirow{2}{*}{ Low } & Cardiology & 1 & 14.2 \\
& Surgery/Cancerology & 1 & 14.2 \\
Others & 5 & 26.3 \\
\hline \multirow{2}{*}{ Intermediate } & Cardiology & 6 & 85.7 \\
& Surgery/Cancerology & 6 & 85.7 \\
& Others & 13 & 68.4 \\
\hline High & Cardiology & 0 & 0 \\
& Surgery/Cancerology & 0 & 0 \\
& Others & 1 & 5.2 \\
\hline
\end{tabular}


In our study, $83.78 \%$ had at least one risk factor for VTE. Of the PIOPED patients [4] 82\% with PE had at least one risk factor for VTE. Prolonged immobilization, age $>60$ years, obesity, pregnancy and postpartum were the risk factors frequently encountered in our series, with respectively $32 \%, 31 \%, 23 \%$ and $20 \%$. Nourelhouda [5] noted that immobilization was the most implicated factor, ahead of surgery and oral contraception.

According to data from the literature, a DVT is authenticated in $3 \%$ of cases for a low score level, $17 \%$ of cases for an intermediate level and $74 \%$ of cases for a high probability level. Raveloson [6] found, according to the Wells score, $95.65 \%$ of their patients with a high clinical probability, $4.35 \%$ with an intermediate clinical probability and no cases with a low probability. Our DVT population had similarities with that of Raveloson [6] since no low probability was found. In the series of Ambid-Lacombe [7], in 40 diagnosed DVT, we found a very low rate of DVT $(0.6 \%)$ for a score less than 0 . The usual strategies for managing suspicions of DVT include the combined use of a clinical probability score and D-dimer assay. Using these strategies, the incidence of DVT in the low risk group is around $3 \%$. Considering that the low-risk group was the group where the diagnosis of DVT was invalidated, there is therefore, according to the literature, a 3\% chance of missing out on DVT if the D-dimer dosage was not carried out. The Ambid-Lacombe [7] population included 48\% of patients for whom D-dimers were not interpretable. In our study, $97.2 \%$ of patients did not benefit from the $\mathrm{D}$-dimer assay.

We deduce that, according to the data of our study, the Wells score with intermediate or high clinical probability, with or without a positive D-dimer assay, is sufficient to evoke the diagnosis of DVT and to make the Venous Doppler echo directly. In other words, the excellent performance of the only used Wells score [8] is, in terms of NPV, equivalent to that of the association of the Wells score and D-dimers. In the PIOPED study [4] which found $28 \%$ of confirmed cases out of 887 patients, where the evaluation of clinical probability was empirical (that is to say without using precise "scores"), $8.3 \%$ of confirmed cases had a low probability, $67.4 \%$ had an intermediate probability and $24.2 \%$ had a high probability. In our series, Wells' low probability rate was much higher at $37.5 \%$, while the revised Geneva rate was $18.75 \%$. Despite the relative superiority of the revised Geneva score over the Wells score for low probability, the risk of misdiagnosis for these two scores would be much too high if the CT chest angiogram was not performed, all the more so as the dosage of D-dimers is hardly performed in our environment.

Taking into account the origin of the patients in our series, the Wells score of the PE showed its excellent performance in the "Surgery/cancerology" subgroup where the low probability was zero, while in the cases with underlying heart disease it was $28.5 \%$. The revised Geneva score of the PE does not discriminate the subgroup of patients with heart disease underlying of that coming from a surgical or oncological setting, since they both have the same low probability proportions (14.2\%) and intermediate (85.7\%). The superiority of the revised Geneva 
score over the Wells score in predicting PE in patients with underlying heart disease was demonstrated by a lower probability ratio, but also by the equality of performance of the revised Geneva score in the two subgroups "Cardiology" and "Surgery/oncology", when we know that these scores correspond better to surgical and oncological settings than to cardiological settings, since these scores did not mention any past history of heart disease or the underlying heart disease that plays an important role in the occurrence of VTE.

\subsection{Study Limitations}

The small number of our population, the absence of a control group, the transversal, descriptive and monocentric nature of our study are all obstacles to objectively evaluating the performance of the scores and their adaptability in our environment.

\subsection{Contribution of Our Study to Knowledge}

- The excellent performance of the Wells score in cardiology.

- The Wells score with intermediate or high clinical probability is sufficient to suggest the diagnosis of DVT and to make the venous Doppler ultrasound directly.

- The revised Geneva score of the EP does not discriminate against patients based on their origin.

- For EP, the use of clinical probability only, predisposes to an increased risk of missing the diagnosis.

- The clinical probability of EP can only be understood within diagnostic strategies that also use objective diagnostic tests.

\section{Conclusion}

As much the excellent performance of the Wells score used alone in the prediction of DVT should give reason to think about alternative strategies for the diagnosis of DVT of the lower limbs that deserve to be evaluated, at least for the patients who cannot benefit from a D-dimer assay, especially for PE, the use of clinical probability alone predisposes to an increased risk of missing the diagnosis. Our study has, moreover, shown that within the population of PE confirmed in our department, more than one patient in 3 according to Wells and nearly one patient in 5 according to Geneva revised had a low probability of presenting an EP. These are all patients who would have gone undiagnosed if objective diagnostic tests had not been performed. This is the reason why the clinical probability of PE can only be understood within diagnostic strategies that also use objective diagnostic tests. Clinical probability therefore does not replace objective tests, and never, alone, makes it possible to make a therapeutic decision.

\section{Authors' Contribution}

All the authors contributed to the conduct of this work. All authors have read 
and approved the latest version of this manuscript.

\section{Conflicts of Interest}

The authors declare no conflict of interest.

\section{References}

[1] Moumneh, T., Penazola, A. and Roy, P.M. (2017) Thrombose veineuse profonde. EMC-Traité de Médecine Akos, 12, 1-6.

[2] Parent, F. and Jovan, R (2017) Embolie pulmonaire: Histoire naturelle, diagnostic, traitement. EMC-Pneumologie, 14, 1-13. https://doi.org/10.1016/S1155-195X(03)00074-4

[3] Samadoulougou, A.K., Millogo, G.R.C., Seghda, T.A.A., Yameogo, R.A., Damoue, S.N.,T., Boro, T., et al. (2015) Performance des scores cliniques de Génève révisé et de Wells dans le diagnostic de l'embolie pulmonaire au Centre hospitalier Universitaire Yalgado Ouédraogo (Burkina Faso). Angéiologie, 67, 52-59.

[4] The PIOPED Investigators (1990) Value of the Ventilation/Perfusion Scan in Acute Pulmonary Embolism. Results of the Prospective Investigation of Pulmonary Embolism Diagnosis (PIOPED). JAMA, 263, 2753-2759. https://doi.org/10.1001/jama.1990.03440200057023

[5] Nourelhouda, C. and Abbassia, D. (2013) Maladie thromboembolique veineuse dans la région de Sidi Bel Abbes, Algérie: Fréquence et facteurs de risque. The Pan African Medical Journal, 16, 45. https://doi.org/10.11604/pamj.2013.16.45.2620

[6] Raveloson, N.E., Vololontiana, M.D. and Rakotoarivony, S. (2011) Aspects épidémiocliniques et évolutives des maladies thrombo-emboliques veineuses à l'Unité de Cardiologie du CHU Antananarivo. Revue d Anesthésie-Réanimation et de Médecine dUrgence, 3, 35-39.

[7] Ambid-Lacombe, C., Cambou, J.P. and Bataille, V. (2009) Excellentes performances du score de Wells et du score de Wells modifié dans le diagnostic de thrombose veineuse profonde proximale ou distale chez des patients hospitalisés ou ambulatoires au CHU de Toulouse: Étude TVP-PREDICT. Journal des Maladies Vasculaires, 34, 211-217. https://doi.org/10.1016/j.jmv.2009.02.004

[8] Wells, P.S. (2007) Integrated Strategies for the Diagnosis of Venous Thromboembolism. Journal of Thrombosis and Haemostasis, 5, 41-50. https://doi.org/10.1111/j.1538-7836.2007.02493.x 